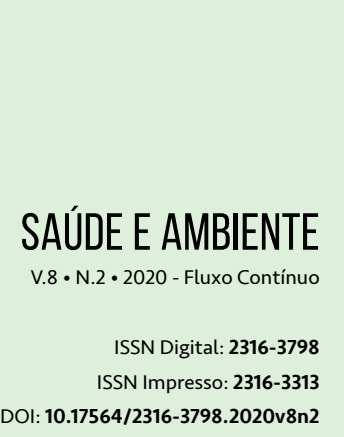

DOI: 10.17564/2316-3798.2020v8n2

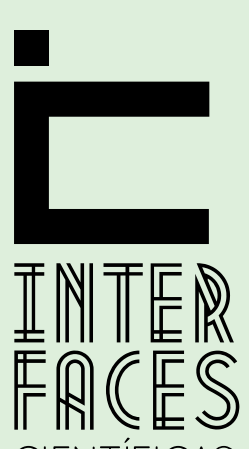

CIENTÍFICAS

\title{
REFLEXÃO SOBRE O ESTADO FíSICO E MENTAL DOS PROFISSIONAIS DE SAÚDE EM ÉPOCA DE COVID-19
}

REFLECTION ON THE PHYSICAL AND MENTAL STATE OF HEALTH PROFESSIONALS AT THE TIME OF COVID-19

REFLEXIÓN SOBRE EL ESTADO FÍSICO Y MENTAL DE LOS PROFESIONALES DE LA SALUD AL MOMENTODE COVID-19

Sonia Oliveira Lima ${ }^{1}$ Manuelli Antunes da Silva ${ }^{2}$ Marina Luzia Duarte Santos ${ }^{3}$ Carla Viviane Freitas de Jesus ${ }^{4}$

\section{RESUMO}

O artigo propõe avaliar o panorama da COVID-19 mediante uma reflexão dos impactos físicos e mentais causados aos profissionais de saúde na sua prática laboral, considerando a complexidade vigente dessa virose que modificou o cotidiano da população. O COVID-19, gerou rápida crise na saúde, economia e na política mundial, sendo declarada em março de 2020, pandemia pela Organização Mundial da Saúde (OMS). Os sistemas de saúde entraram em colapso, causando uma tragédia na humanidade, necessitando de estratégias rápidas pelo setor governamental. Os profissionais que detêm o conhecimento científico, assumem uma posição diferenciada, por serem responsáveis pela determinação de ações de cuidados a saúde. A tensão crônica vivenciada por estes trabalhadores pode provocar exaustão emocional, despersonalização e baixa realização pessoal, responsáveis por emoções e cognições negativas, levando a comportamentos que reduzem o sistema imunológico. Estes especialistas, embora consciente dos riscos, por não possuir condições econômica e/ou emocional de poder usufruir de um lugar exclusivo nesse período de isolamento social, arrisca a saúde de outros e de seus entes queridos. Por estar na linha de frente nessa pandemia, é considerado um herói pela mídia e população, porém se sente estigmatizado pelos moradores próximos e da sua residência devido ao medo da contaminação. As condições geradas pelo COVID-19 são fatores estressores suficientes para suscitar traumas físicos e psicológicos nestes trabalhadores, sendo comparadas a desastres naturais e guerras. São necessárias medidas para melhoria no ambiente de trabalho, estimulando o desempenho laboral e a capacidade de lidar diariamente com a doença, a morte e o sofrimento da comunidade. 


\section{PALAVRAS-CHAVE}

Pandemias. Infecções por Coronavírus. Pessoal de Saúde.

\section{ABSTRACT}

The article proposes to evaluate the panorama of COVID-19 through a reflection of the physical and mental impacts caused to health professionals in their work practice, considering the current complexity of this virus that has changed the daily life of the population. COVID-19, generated a rapid crisis in health, economy and world politics, being declared in March 2020, a pandemic by the WHO. Health systems have collapsed, causing a tragedy for humanity, requiring rapid strategies by the government sector. The professionals who have scientific knowledge, assume a different position, as they are responsible for determining health care actions. The chronic tension experienced by these workers can cause emotional exhaustion, depersonalization and low personal fulfillment, responsible for negative emotions and cognitions, leading to behaviors that reduce the immune system. These specialists, although aware of the risks, for not having the economic and / or emotional conditions to be able to enjoy an exclusive place in this period of social isolation, risk the health of others and their loved ones. For being at the forefront of this pandemic, he is considered a hero by the media and the population, but he feels stigmatized by the nearby residents and his home due to fear of contamination. The conditions generated by COVID-19 are sufficient stressors to cause physical and psychological trauma in these workers, being compared to natural disasters and wars. Measures are needed to improve the work environment, stimulating work performance and the ability to deal daily with the community's illness, death and suffering.

\section{KEYWORDS}

Pandemics. Coronavirus Infections. Health Personnel.

\section{RESUMEN}

El artículo propone evaluar el panorama de COVID-19 a través de una reflexión de los impactos físicos y mentales causados a los profesionales de la salud en su práctica laboral, considerando la complejidad actual de este virus que ha cambiado la vida diaria de la población. COVID-19 generó una rápida crisis en salud, economía y política mundial, siendo declarada en marzo de 2020 una pandemia por la OMS. Los 
sistemas de salud se han derrumbado, causando una tragedia para la humanidad, que requiere estrategias rápidas por parte del sector gubernamental. Los profesionales que tienen conocimiento científico, asumen una posición diferente, ya que son responsables de determinar las acciones de atención médica. La tensión crónica experimentada por estos trabajadores puede causar agotamiento emocional, despersonalización y baja realización personal, responsables de las emociones y cogniciones negativas, lo que lleva a comportamientos que reducen el sistema inmunológico. Estos especialistas, aunque conscientes de los riesgos, por no tener las condiciones económicas y/o emocionales para poder disfrutar de un lugar exclusivo en este período de aislamiento social, arriesgan la salud de los demás y de sus seres queridos. Por estar a la vanguardia de esta pandemia, los medios de comunicación y la población lo consideran un héroe, pero se siente estigmatizado por los residentes cercanos y su hogar debido al temor a la contaminación. Las condiciones generadas por COVID-19 son factores estresantes suficientes para causar traumas físicos y psicológicos en estos trabajadores, en comparación con los desastres naturales y las guerras. Se necesitan medidas para mejorar el ambiente de trabajo, estimular el desempeño laboral y la capacidad de lidiar diariamente con la enfermedad, la muerte y el sufrimiento de la comunidad.

\section{PALABRAS CLAVE}

Pandemias. Infecciones por coronavirus. Profesional de Salud.

\section{INTRODUCÇÃO}

No cenário das práticas de combate à pandemia da COVID-19, deve-se considerar uma complexidade que envolve aspectos políticos, sociais, educativos, econômicos e de saúde. Os especialistas envolvidos se empenham no controle da pandemia, além de tentar alcançar as normalidades fisiológica e econômica dos sujeitos envolvidos. Nessa relação, os profissionais que detêm o conhecimento científico, assumem uma posição diferenciada, por serem responsáveis pela determinação de ações de cuidados a saúde (KURCGANT et al., 2006).

A infecção pela COVID-19 ocorreu de maneira explosiva na maior parte do mundo, por meio de secreções respiratórias, com período de incubação que varia de um a quatorze dias (SINGHAL, 2020). Embora a letalidade dessa infecção seja baixa, a sua capacidade de transmissão é muito alta, o que aumenta a mortalidade em um curto espaço de tempo (FARIAS, 2020).

Medidas governamentais foram tomadas com o intuito de minimizar os danos decorrentes da contaminação em massa, entre elas o fechamento de serviços não-essenciais, o distanciamento social, evitando aglomerações a fim de manter uma distância mínima de um metro e meio para outras pessoas. Para reduzir a contaminação foi também adotada a obrigatoriedade de quarentena por quatorze dias para os suspeitos de infecção e a determinação do isolamento social para a população, sendo orientadas a permanecerem em domicílio principalmente pessoas com comorbidades e idosos (BRASIL, 2020). 
Devido à falta de estrutura e de profissionais para o atendimento dos infectados, outras medidas foram adotadas, como construção de hospitais de campanha, antecipar a formatura de profissionais e requisitar acadêmicos de saúde para atuarem na linha de frente. Essas estratégias foram necessárias em virtude da complexidade dos sintomas e da precariedade dos serviços de saúde (BAPTISTA; FERNANDES, 2020). É importante destacar que os trabalhadores da saúde que estão atuando na linha de frente são mais susceptíveis de serem contaminados (LAl et al., 2020).

Este profissional tem conhecimento de que mesmo contaminado pode não ter sintomas, no período de incubação pode transmitir a infecção e quando sintomático ter o risco de culminar com a Síndrome Respiratória Severa Aguda (SARS-CoV-2), falência de órgãos e morte (WANG et al., 2020). Portanto, além do acometimento físico causado pelo coronavírus, a saúde mental dessa população está sendo afetada. Os indivíduos em isolamento vivenciam momentos de estresse com a perda de contato íntimo e social que culminam com sensações de solidão ou raiva. No entanto, as consequências psicológicas tendem a ser subestimadas e, muitas vezes, negligenciadas, acarretando assim, em estratégias falhas no combate à doença (ORNELL et al., 2020).

Os danos da saúde mental são mais duradouros que o tempo da própria pandemia (ORNELL et al., 2020). Os efeitos adversos que acometem os profissionais expostos a tensão e estresse intenso afetam a sua qualidade de vida, gerando problemas de insatisfação e exaustão, o que pode interferir negativamente na qualidade dos serviços prestados.

Quando os profissionais não encontram no trabalho a realização esperada pode desenvolver a síndrome de esgotamento profissional, conhecida como síndrome de burnout. Esta síndrome é reconhecida mundialmente como um dos grandes problemas psicossociais que afeta principalmente profissionais envolvidos no cuidado à saúde, educação e serviços humanos, gerando uma importante questão ocupacional e social (VIEIRA, 2010; LIMA et al., 2013).

Assim, esse artigo propõe avaliar o panorama da COVID-19 mediante uma reflexão sobre os impactos físicos e mentais causados aos profissionais de saúde na sua prática laboral, considerando a complexidade vigente de uma virose que modificou o cotidiano da população mundial.

\section{ASPECTOS DA PANDEMIA DE COVID-19}

O coronavírus 2019 (SARS-CoV-2), causador da COVID-19, gerou uma rápida crise na saúde, na economia e na política mundial, sendo declarada como pandemia pela Organização Mundial da Saúde (OMS) em 11 de março de 2020. Em 8 de maio de 2020 havia 3.935.764 de casos da COVID-19 e 274.651 de óbitos nos 187 países afetados (WHO, 2020a). Os sistemas de saúde desses países entraram em colapso, causando uma tragédia na humanidade, necessitando de empenho e estratégias rápidas pelo setor governamental.

Os primeiros casos foram identificados em dezembro de 2019, em Wuhan, província da Hubei na China, onde ocorreu um surto caracterizado por uma pneumonia infecciosa aguda (BAO et al., 2020). Em março de 2020 houve o pico de notificações de novos casos e o maior número de mortos, 
nesse período, o vírus já havia sido notificado em outros países, sendo considerado uma pandemia pela OMS (HUANG; ZHAO, 2020). A maioria dos países com presença da infecção iniciou a redução de deslocamento vertical enquanto alguns países já haviam adotado medidas mais rigorosas com fechamento de fronteiras, distanciamento e isolamento social.

Por se tratar de uma infecção respiratória aguda, o vírus pode ser transmitido de humano para humano (transmissão direta) sendo conhecida como um dos principais mecanismos de infecção, ou por transmissão indireta por meio de superfícies contaminadas. O tempo de sobrevida que o SARS-CoV-2 permanece infeccioso e viável depende do local, da quantidade da secreção eliminada pelo paciente e da superfície depositada.

$O$ vírus é perigoso devido a velocidade de disseminação e por ser resistente ao ambiente externo (BRITO et al., 2020). A OMS recomenda lavagem e/ou antissepsia frequente das mãos, uso de máscaras, quarentena, distanciamento e isolamento social, com intuito de reduzir a transmissão e, o consequente, aumento dos casos (WHO, 2020b). Essa recomendação tem o objetivo de tentar evitar o crescimento em um curto espaço de tempo, superlotando os serviços de saúde que se mostrou insuficiente para atender ao pico dessa pandemia.

O indivíduo acometido pela COVID-19 pode ser assintomático ou apresentar sintomas como febre, tosse, dor de garganta, dispneia, mialgia, diarreia, podendo chegar a Síndrome Respiratória Severa Aguda, falência de órgãos e morte (WANG et al., 2020). As manifestações clínicas variam em crianças, adolescentes, adultos e idosos, sendo os sintomas mais frequentes relatados, febre (88,7\%), tosse $(67,8 \%)$ e fadiga $(38,1 \%)$ (GUAN et al., 2020). Os pacientes em estado grave apresentam sinais e sintomas relacionados à insuficiência respiratória, como dispneia, baixos sons respiratórios, embotamento à percussão, elevação e diminuição do tremor da fala à palpação do tórax (JIN et al., 2020).

Também pode-se encontrar alterações aos exames de imagens, estudos apresentam como escoIha a tomografia computadorizada do tórax que mostra uma imagem semelhante a vidro fosco (GUAN et al., 2020; ZHANG et al., 2020). A maioria dos infectados pelo coronavírus são assintomáticos ou apresentam sintomas leves que podem ser confundidos com outras doenças como crises respiratórias gripais, alérgicas ou asmáticas, infecção intestinal, tensão emocional com astenia. Em virtude do medo de estar com a COVID-19, desde que é divulgado constantemente o número de óbitos por essa afecção, o indivíduo pode entrar em desespero e buscar os serviços de saúde.

A falta de uma terapia eficaz faz com que o tratamento da COVID-19 dependa das características resultantes dessa infecção, sendo baseado no controle dos sintomas e oferta de suporte ventilatório, o que movimentou a comunidade científica em busca de vacina e de tratamento específico (GUO et al., 2020; ZHANG et al., 2020). A OMS em parceria com mais de 100 países, busca avaliar a eficácia de quatro medicamentos diferentes, sendo eles remdesivir, cloroquina e hidroxicloroquina, lopinavir/ritonavir e interferon beta1a, objetivando identificar qual opção atrasa a progressão da doença e melhora a sobrevida do paciente (WHO, 2020b). 0 resultado destas medidas reflete na taxa de letalidade e de mortalidade desse agravo.

Estudos de imunopatologia de afecções causadas por SARS-CoV-2 ainda estão sendo investigados globalmente (KUMAR et al., 2020). Observou-se, porém, que a cepa do vírus altera o sistema imu- 
nológico quando uma pessoa é infectada, portanto é importante que a população predisposta a um distúrbio imunológico, se mantenha isolada.

Devido ao pânico generalizado e constante preocupação, o surto por COVID-19 acarretou além dos distúrbios físico, disfunções psíquicas, fator de redução da imunidade. 0 impacto psicológico, acontece quando se instala o sentimento de adoecer ou morrer, além de desamparo e estigma proporcionado pela infecção (BAO et al., 2020). Além disso, a incerteza quanto ao futuro é exacerbada com mitos e informações fraudulentas (WANG et al., 2020).

Torna-se imprescindível o controle de notícias negativas, que proporciona o pânico, e de falsas informações. Presença de sintomas semelhantes aos divulgados pela infecção do SARS-CoV-2, faz que seu portador se dirija aos serviços de saúde, o que provoca a sua superlotação. De maneira oposta, doenças que necessitam de atendimento médico, são negligenciadas por medo da contaminação nos serviços de urgência, isso é perceptível pelo aumento de óbitos nesse período decorrentes de complicações de afecções cardiovasculares, neurológicas, endócrinas e outras.

\section{ATUAÇÃO DOS PROFISSIONAIS DIANTE DO COVID-19}

A comunidade científica mundial tem trabalhado conjunta e continuamente para entender os aspectos epidemiológicos, clínicos e o tratamento da COVID-19. Essa prática unificou o mundo no momento de isolamento social, onde aspectos econômicos e psicológicos de grande magnitude reflete sobre o estado físico e mental de profissionais de várias áreas, em particular dos profissionais de saúde.

Os trabalhadores da saúde portador de conhecimento sobre a gravidade da COVID-19 e da importância do distanciamento e isolamento social são convocados a atuar na assistência a enfermos com possibilidade dessa infecção. É nesse sentido que ao praticar o seu labor esses profissionais necessitam sair do seu isolamento e romper o distanciamento para atender a comunidade dentro de diversos cenários. É necessário o conhecimento das práticas de uso de equipamentos de proteção individual (EPI), onde alguns desses, principalmente recém egressos das universidades, ainda são pouco familiarizados com o uso correto dos EPI.

No enfrentamento dessa infecção medidas preventivas devem ser implantadas, tais como, realização de atividades de educação permanente, maior aproveitamento de equipamentos e tecnologias, adoção de pausas esporádicas durante a jornada de trabalho, atenção ao clima organizacional para uma boa governança dos conflitos decorrentes dos posicionamentos intra e inter equipes, de tal forma a garantir ambientes de trabalho geradores de saúde física e mental (SILVA et al., 2015). Dessa maneira, os trabalhadores ao se sentirem apoiados poderão prestar melhor assistência aos usuários.

Sintomas físicos da COVID-19, como febre, tosse, dispneia, podem ocorrer em pacientes não infectados, devido a outras doenças ou a aspectos emocionais (WHO, 2020a). O conhecimento é adquirido e inseparável das práticas o que evidencia a estreita relação entre ambos (VELLOSO et al., 2010). É a atitude crítica diante da vivência que permitirá um diagnóstico acertado de determinada doença, com possibilidade de agir adequadamente no tratamento. Portanto, o tempo de exercício 
da profissão qualifica a assistência no vasto cenário da atenção à saúde, onde a prática confere inteligibilidade para percepção de diagnósticos diferenciais, o que aumenta o nível de estresse dos profissionais, em especial, do recém-formado.

Verificou-se, em estudo populacional na China, que a pandemia da COVID-19, por afetar o estado mental decorrente do isolamento, impactos econômicos e óbitos, induziu a um maior consumo de álcool e outras drogas. A classe médica destacou-se no uso dessas drogas e apresentou maior incidência de ansiedade, depressão, insônia e estresse que a população geral. Na tentativa de minimizar esse impacto negativo, foram criadas plataformas on-line oferecidas por profissionais da saúde mental para ajuda psicológica tanto aos pacientes quanto aos profissionais de saúde e para população não infectada (AHMEDA et al., 2020). Muitos países seguiram a iniciativa chinesa e no Brasil esse canal de atendimento prioriza os profissionais de saúde.

0 trabalhador de saúde ao exercer sua função assume as consequências diante de pacientes críticos onde seu posicionamento e postura serão avaliados pela comunidade. Esses profissionais, por vezes, se veem em situações de escolha de que paciente tem prioridade de atendimento, enquanto policiados pelos familiares, colegas, gestores, imprensa e órgãos judiciais. Assim, entende-se as vastas tensões inerentes tanto do contexto físico quanto mental, proveniente dos riscos eminentes da infecção decorrente de uma contaminação pela COVID-19. A necessidade de algumas vezes ultrapassar a carga horária devida, ao assumir o seu exercício, esse profissional, pode se tornar um vetor de contaminação.

A tensão crônica vivenciada por estes trabalhadores pode provocar exaustão emocional, despersonalização e baixa realização pessoal, por não corresponder a expectativa, caracterizando a possibilidade da síndrome de burnout (SILVA et al., 2015). As consequências geradas pelos fatores estressantes, tanto físico como relacionados a imprevisibilidade de uma contaminação pela COVID-19 podem ser responsáveis por emoções e cognições negativas, levando a comportamentos que culminam com a redução do sistema imunológico.

Estes especialistas, embora consciente dos riscos, por não possuir condições econômica e/ou emocional de poder usufruir de um lugar privilegiado e exclusivo nesse período de isolamento social preconizado como fator importante de prevenção e controle da COVID-19, arrisca a saúde de outros e de seus entes queridos. Por estar na linha de frente do atendimento, nessa pandemia, é considerado um herói pela mídia e população, porém se sente estigmatizado pelos moradores próximos e da sua residência devido ao medo da contaminação.

\section{CONCLUSÃO}

A carga horária de trabalho excessiva, a insatisfação pessoal, pode ocasionar um processo de adoecimento com ameaça ao bem-estar dos profissionais, refletindo no atendimento à população. As condições geradas pela pandemia da COVID-19 são fatores estressores suficientes para suscitar traumas físicos e psicológicos nestes trabalhadores, sendo comparadas a desastres naturais e guerras.

0 que denota a importância da implementação de medidas preventivas e interventivas com o intuito da melhoria no ambiente de trabalho, estimulando o desempenho laboral e a capacidade de 
lidar diariamente com a doença, a morte e o sofrimento da comunidade, obedecendo as normas estabelecidas pela OMS.

\section{REFERÊNCIAS}

AHMEDA, M. Z. et al. Epidemic of COVID-19 in China and associated Psychological Problems. Asian J. Psychiatr., v. 51, p. 1-7, 2020.

BAO, Y. et al. 2019-nCoV epidemic: address mental health care to empower society. The Lancet., v. 395, n. 10224, p. 37-38, 2020.

BAPTISTA, A. B.; FERNANDES, L. V. Covid-19, análise das estratégias de prevenção, cuidados e complicações sintomáticas. Desafios - Rev. Interdiscipl. UFT, v. 7, n. esp-3, p. 38-47, 2020.

BRASIL. Ministério da Saúde. Como é definido um caso suspeito de coronavírus? Brasília: MS. 2020. Disponível em: https://coronavirus.saude.gov.br/sobre-a-doenca\#tratamento. Acesso em: 6 mai 2020.

BRITO, S. B. P. et al. Pandemia da COVID-19: o maior desafio do século XXI. Vigil. Sanit. Debate, v. 8, n. 2, maio 2020.

FARIAS, H. S. O avanço da Covid-19 e o isolamento social como estratégia para redução da vulnerabilidade. Esp. Econ., v. 17, p. 1-12, 2020.

GUAN, W. et al. Clinical characteristics of coronavirus disease 2019 in China. N. England J. Med., v. 382, n. 18, p. 1708-1720, 2020.

GUO, Y.R. et al. The origin, transmission and clinical therapies on coronavirus disease 2019 (COVID-19) outbreak - an update on the status. Mil. Med. Res., v. 7, n. 1, p. 11, 2020.

HUANG, Y.; ZHAO, N. Generalized anxiety disorder, depressive symptoms and sleep quality during COVID-19 outbreak in China: a web-based cross-sectional survey. Psychiatr. Res., v. 288, p. 1-6, 2020.

JIN, Y. H. et al. A rapid advice guideline for the diagnosis and treatment of 2019 novel coronavirus (2019-nCOV) infect pneumonia (standard version). Mil. Med. Res., v. 7, n. 1, p. 4, 2020.

KUMAR, S. et al. Host immune response and immunobiology of human SARS-CoV-2 infection. In: Saxena, S.K. (Ed.) Coronavirus Disease 2019 (COVID-19). Chan: Springer, 2020. 
KURCGANT, P. et al. O planejamento nas organizações de saúde: análise da visão sistêmica. Rev. Gaúcha Enferm., v. 27, n. 3, p. 351-355, 2006.

LAI, J. et al. Factors associated with mental health outcomes among health care workers exposed to coronavirus disease 2019. JAMA Netw Open, v. 3, n. 3, p. 1-12, 2020.

LIMA, R. A. S. et al. Vulnerabilidade ao burnout entre médicos de hospital público do Recife. Ciên. Saúde Col., v. 18, p. 1051-1058, 2013.

ORNELL, F. et al. "Pandemic fear" and COVID-19: mental health burden and strategies. Braz. J. Psychiatr., v. 42, n. 3, p. 232-235, 2020

SILVA, S. C. P. S. et al. A síndrome de burnout em profissionais da Rede de Atenção Primária à Saúde de Aracaju, Brasil. Ciên. Saúde Col., v. 20, p. 3011-3020, 2015

SINGHAL T. A review of coronavirus disease-2019 (COVID-19). Indian J. Pediatr., v. 87, p. 281-6, 2020.

VELLOSO, I. S. C. et al. Reflexões sobre relações de poder na prática de enfermagem. Rev. Gaúcha Enferm., v. 31, n. 2, p. 388-391, 2010.

VIEIRA, I. Conceito(s) de burnout: questões atuais da pesquisa e a contribuição da clínica. Rev. Bras. Saúde Ocup., v. 35, n. 122, p. 269-276, 2010.

WANG, C. et al. Immediate psychological responses and associated factors during the initial stage of the 2019 Coronavirus Disease (COVID-19) epidemic among the general population in China. Int. J.

Environ. Res. Publ. Health, v. 17, n. 5, p. 1-25, 2020.

WHO - World Health Organization. Coronavirus disease (COVID-19) outbreak. Geneva: World Health Organization; 2020a. Disponível em: www.who.int/emergencies/diseases/novelcoronavirus-2019. Acesso em: 8 maio 2020.

WHO - World Health Organization. “Solidarity” clinical trial for COVID-19 treatments. Geneva: World Health Organization; 2020b. Disponível em: www.who.int/emergencies/diseases/novelcoronavirus-2019/global-research-on-novel-coronavirus-2019-ncov/solidarity-clinical-trial-forcovid-19-treatments. Acesso em: 6 maio 2020.

ZHANG, J. et al. Therapeutic and triage strategies for 2019 novel coronavirus disease in fever clinics. Lancet Resp. Med., v. 8, n. 3, p. e11-e12, 2020. 


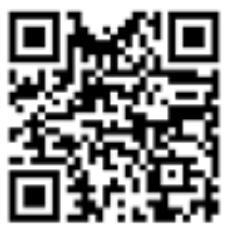

A autenticidade desse artigo pode ser conferida no site https://periodicos. set.edu.br

1 Doutora em Clínica Cirúrgica; Médica; Professora permanente do programa Saúde e Ambiente, e do curso de medicina da Universidade Tiradentes - UNIT.

E-Mail: sonialima.cirurgia@gmail.com

2 Acadêmica do Curso de Medicina da Universidade Tiradentes - UNIT. E-mail: manuellibrito@hotmail.com.

3 Acadêmica do Curso de Medicina da Universidade Tiradentes - UNIT. E-mail: marina.duarte7@hotmail.com

4 Graduada em Enfermagem; Doutoranda do Programa de Pós-graduação em Saúde e Ambiente da Universidade Tiradentes - UNIT. E-Mail: carlavfj@gmail.com

\section{@( $\odot \odot$}

Este artigo é licenciado na modalidade acesso abertosob a Atribuição-Compartilhalgual CC BY-SA

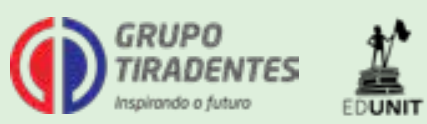

